\title{
PERFORATION OF THE BOWEL IN HENOCH-SCHÖNLEIN PURPURA
}

\author{
BY \\ R. BASU \\ From the Alder Hey Children's Hospital, Liverpool
}

(RECEIVED FOR PUBLICATION FEBRUARY 20, 1959)

Perforation of the bowel in Henoch-Schönlein purpura has rarely been recorded in the literature. Two instances of this catastrophe are recorded here.

\section{Case Reports}

Case 1. D.J.S., a $4 \frac{1}{2}$-year-old boy, was admitted to the medical wards of Alder Hey Children's Hospital with a diagnosis of Henoch-Schönlein purpura. On the tenth day the child showed signs of intestinal obstruction and a mass was palpable in the right iliac fossa. He was transferred to the surgical wards with a diagnosis of intussusception.

At operation an ileo-ileal intussusception was reduced with some difficulty. The bowel was viable but there was some subserosal haemorrhage. The immediate post-operative period was very satisfactory, but, on the fifth post-operative day, the patient suddenly collapsed and on examination had signs of generalized peritonitis. After adequate intravenous therapy he was taken to the theatre.

At the second operation, on opening the peritoneal cavity, gas and faecal material were encountered. Two inches of the ileum were gangrenous and numerous large perforations were found in the healthy segment of gut, one foot proximal to the intussuscepted area. Resection of the gangrenous segment was carried out and the two ends of the bowel were exteriorized, as the condition of the patient did not permit an anastomosis. Postoperatively he was critically ill and died four days later.

Autopsy. Oesophagus: The mucosa of the lower third showed extensive ulceration but the muscle coat was intact.

Stomach: There was a rupture along the greater curve and posterior surface with escape of stomach contents into the peritoneal cavity. The edges of the rupture were soft and in parts congested. The rupture had certainly not been present for long before death.

Intestine: The intestine, above and below the ileostomy, was congested on the serosal surface and was oedematous throughout. There were small areas with petechial haemorrhages scattered throughout the whole length but no gross lesions were detected.

Spleen and kidney: These showed intense capillary congestion.
Lungs: They were moderately collapsed. Haemorrhagic areas were seen on the surface of all lobes and ante-mortem thrombi found in the radicles of the pulmonary arteries on both sides. There were multiple areas of infarction.

Histology. The specimen of resected ileum consisted of approximately $30 \mathrm{~cm}$. of ileum. The whole length of the gut was intensely injected with a patchy purulent exudate over the surface. There were numerous ragged perforations at various sites.

Microscopical examination of a section of the ileum, away from the perforations, showed an inflammatory serosal and subserosal reaction with congestion. At the edge of a perforation, the wall of the intestine showed haemorrhagic necrosis only.

The diagnosis was Henoch-Schönlein purpura perforation of the gut.

Case 2. T.R., a boy aged $8 \frac{1}{2}$ years, was admitted to the medical wards of an outlying hospital, with a diagnosis of Henoch-Schönlein purpura. He was treated with prednisolone $5 \mathrm{mg}$. twice a day. On the tenth day the abdomen started to distend and two days later signs of peritonitis developed. A straight radiograph of the abdomen revealed fluid levels. He was transferred to the surgical wards of Alder Hey Children's Hospital and at laparotomy a curious state of affairs was seen.

The peritoneal cavity could not be entered as the 'bowel' was adherent to the peritoneum. After careful dissection, the bowel was found to have three large perforations and was bound together, by adhesions, in one inextricable mass. I say 'bowel' because it was not possible to differentiate large from small gut. The three perforations were closed. A catheter was inserted into what one thought to be the proximal segment of gut and brought out through a separate incision.

After the operation the child was kept on continuous intravenous therapy and gastric suction for 15 days. His general condition remained fair but his state $o$ nutrition suffered considerably. Ileus persisted and from the fifteenth post-operative day onwards Aminosol was administered through a polythene catheter which had been advanced into the inferior vena cava through the femoral vein. In spite of all care the patient died on the thirtieth post-operative day. 
Autopsy. Heart and blood vessels: The right auricle and ventricle were distended. The mitral valve cusp had large adherent vegetations which appeared to be of recent origin. The inferior vena cava, below the renal veins, contained infected thrombi and so did the femoral and iliac veins.

Alimentary passages and peritoneum: The small bowel was matted together by adhesions. About $2 \mathrm{ft}$. away from the termination of the ileum, at the site of two sutured perforations, the adhesions were very dense and a further perforation was present. The gut appeared to be obstructed being grossly dilated proximal to this point and collapsed distally.

Brain: There was an early abscess in the substance, with an early pyogenic meningitis, of embolic origin probably.

Lungs: Some branches of the vessels contained antemortem clot, probably embolic in origin.

Kidney: There was gross pyaemic infarction with large abscesses in many areas. These grew Gram + ve cocci.

\section{Discussion}

Wolfsohn (1947) quotes Willan as having descriked visceral crises in purpura as early as 1808 , thus ante-dating by 66 years Henoch's classical paper.

Henoch-Schönlein purpura, also known as allergic purpura, is characterized by: (1) Cutaneous manifestations, pleomorphic in character, involving mainly the extensor aspects of the limbs. (2) Colicky abdominal pain, vomiting, intestinal bleeding and intussusception. (3) Pain in the joints, haemarthrosis.

Other complications listed (Ackroyd, 1953) are (4) Bleeding from the gums and epistaxis. (5) Intracranial haemorrhages producing convulsions. (6) Nephritis, indistinguishable from Ellis' Type I nephritis, causing haematuria.

In this paper only the question of perforation of the bowel will be discussed. This complication has been seen only very occasionally. Ballin and Morse (1930) reported a case, an adult male with Henoch's purpura, in which perforation of the bowel occurred a few months after reduction of an intussusception. Unfortunately this case also died. Goldstein (1928) refers to haemorrhagic ulcers in the wall of the gut of patients with Henoch's purpura and considers that these perforate on very rare occasions.
Pathology

Kreidberg, Dameshek and Latorraca (1955) describe the lesions of Henoch-Schönlein purpura as acute perivasculitis in the walls of the small vessels occurring as a result of an immunologic mechanism. This was also observed in the two reported cases.

It is suggested that an acute vascular reaction, of this nature, in the small vessels could devitalize the wall of the bowel at several points and thus predispose to perforation. Nevertheless it is surprising, though fortunate, that this complication is so rarely seen. It must be presumed then that the reaction is not sufficiently severe to cause devitalization of the bowel wall.

Intussusception is the commonest surgical complication of this 'syndrome'. It requires prompt diagnosis and operation. Such an intussusception, if left untreated, may perforate and cause peritonitis due to the presence of non-viable gut. This also holds true to gangrenous bowel of any origin. But perforation of the bowel, as we have seen in these two cases, without any such cause is quite a separate entity. In Case 1 perforation of the bowel occurred in a healthy segment. Unfortunately Case 2 was complicated by bacterial endocarditis, due to infected emboli, and in addition had received prednisolone preoperatively. As perforation is not very common with this drug and as the endocarditis was postoperative, we can safely assume that this was another case of perforation per se.

\section{Summary}

Two cases of intestinal perforation, complicating Henoch-Schönlein purpura, are described.

I am grateful to Miss Isabella Forshall and to $\mathrm{Mr}$. P. P. Rickham for permission to publish their cases and also for their help in the preparation of this paper. My thanks are also due to Dr. E. G. Hall for the post-mortem and pathological findings.

\section{REFERENCES}

Ackroyd, J. F. (1953). Amer. J. Med., 14, 605.

Ballin, M. and Morse, P. F. (1930). Ann. Surg., 91, 711.

Goldstein, E. (1928). Med. Clin. N. Amer., 12, No. 3 (New York Number), 869.

Kreidberg, M. B., Dameshek, W. and Latorraca, R. (1955). New Engl.J. Med., 253, 1014 .

Wolfsohn, H. (1947). Arch. Dis. Childh., 22, 242. 